relative amounts of methylation of the two mature molecules are different, so that methylation could be used to identify the parts of the $45 \mathrm{~S}$ precursor carrying these sequences. The experimental problem is to obtain identified fragments of the $45 \mathrm{~S}$ molecule. Liau and Hurlbert generated suitable conditions by incubating Novikoff rat tumour nucleoli in the absence of nucleotides; this prevents elongation of polynucleotide chains, generating a series of incomplete precursor molecules of varying lengths starting from the $5^{\prime}$ end, which are methylated in vitro. The $18 \mathrm{~S}$ methylation pattern is found in the shorter transcriptions and the $28 \mathrm{~S}$ pattern is seen in the longer molecules.

One of the most satisfying conclusions to be drawn is that the same relative organisation of mature rRNA sequences within the precursor is seen in bacterial and eukaryotic systems. The smaller molecule precedes the larger, although more extensive spacer sequences have evolved in the eukaryotic systems. It seems clear that the relative locations of the $18 \mathrm{~S}$ and $28 \mathrm{~S}$ RNA sequences seen in the HeLa and Xenopus precursors by electron microscopy are indeed correct; but the polarity is reversed. The explanation seems to lie in the use of the exonuclease to determine the polarity of the RNA; clearly in the system used, the $5^{\prime}$ rather than the $3^{\prime}$ end must have been digested, perhaps by some contaminating enzyme.

What is the function of the spacer regions in the common precursor? Little information is available on the extensive spacers of the eukaryotic precursor, but the striking observation that a tRNA gene lies between the $16 \mathrm{~S}$ and $23 \mathrm{~S}$ sequences in an E.coli unit has been reported by Lund et al. (Cell, 7, 165-177; 1976). The E.coli genome possesses several copies of the genes for rRNA, organised in transcription units that display the sequence 16S-23S-5S RNA (with the assignment of the 5S RNA to the end of the unit being less certain than the tandem arrangement of the $16 \mathrm{~S}$ and $23 \mathrm{~S}$ sequences). The transducing phage $\lambda$ rif $^{d} 18$ carries one of the sets of the genes for $16 \mathrm{~S}$ and $23 \mathrm{~S} \mathrm{RNA}$, as well as genes for some RNA polymerase subunits, ribosomal proteins, and a protein synthesis factor. When cells are infected with this phage, the synthesis of two small RNA species is greatly stimulated: one is 5S RNA and the other is $\mathrm{tRNA}_{2}{ }^{\text {Glu }}$. Where is the tRNA gene located? By showing that a radioactively labelled $\mathrm{tRNA}_{2}{ }^{\text {Glu }}$ molecule could hybridise to a phage DNA deleted for the 23S sequence, but could not hybridise to a phage DNA where the deletion extended from the $23 \mathrm{~S}$ sequence into the $16 \mathrm{~S}$ sequence, they

\title{
The Red Queen lives
}

from Leigh Van Valen

WHEN the Red Queen heard of her reported dethronement (Hallam, Nature, 259, 12-13; 1976), she smiled from her throne and said, "As Mark Twain remarked on a report of his death, I find the report somewhat exaggerated".

One side of a controversy is often convincing until the other side appears. Hallam primarily based his conclusion on two recent papers of Salthe and Sepkoski; my response to these has not yet been published and will be part of a broader discussion. For now I summarise my comments on the points Hallam discussed.

There are several possible hypotheses to explain the law of constant extinction, which produces a linearity of the survivorship curves of adaptively similar groups on a semilog plot. One is the Red Queen's Hypothesis, that the sum of absolute fitnesses in a community is constant. Another, used by Raup et al. (J. Geol., 81, [for 1973], 525-543; 1974) in their simulations and which I call the Fieldof-Bullets Hypothesis, is that the probability of extinction is constant for all groups at all times. This hypothesis is unlikely (Van Valen, Evol. Theory, 1, 1-30; 1973; Nature, 257, 515-516; 1975). A third hypothesis, which Salthe (Paleobiology, 1, 356358 ; 1975) proposed and which may be called the Hypothesis of Triviality, is that any reasonable pattern of causation leads to near linearity and so no specific explanation is appropriate. There are several problems with the Hypothesis of Triviality, but it would seem to be disproved by the existence of a few systematically nonlinear survivorship curves. That such deviations from linearity have known no possible particular explanations

were able to conclude that the gene for the tRNA must lie between the genes for the $16 \mathrm{~S}$ and $23 \mathrm{~S}$ RNAs (Yamamoto, Lindahl and Nomura, Cell, 7, 179-190; 1976). This location was confirmed by Wu and Davidson (Proc. natn. Acad. Sci. U.S.A., 72, 4506-4510; 1975). in experiments utilising a new technique for visualising RNA-DNA duplex regions formed along a single strand of DNA.

An obvious question is whether this form of organisation is seen in all the $16 \mathrm{~S}-23 \mathrm{~S}$ transcription units of the bacterial genome. Lund et al. (op. cit.) examined the transducing phage ๑80 if $^{\mathrm{r}}$, which also carries an rRNA transcription unit, and showed that it is homologous with the $\lambda r i f^{\mathrm{d}} 18$ phage over most of the bacterial sequences
(Van Valen, Evol. Theory, 1, 1-30; 1973) merely accentuates their reality. Simpson (Tempo and Mode in Evolution; 1944) used a phenomenon equivalent to part of this exceptional non-linearity as the basis for distinguishing what he called bradytely and horotely as more or less discrete evolutionary modes. Horotely is intimately related to linearity or survivorship curves.

Sepkoski (Paleobiology, 1, 343-355; 1975 ) in fact claims that most of the curves are non-linear and that the distribution of the lengths of stratigraphic units produces a bias that gives spurious linearity. However, the main result of this bias, when I apply my methods to his simulations, is a reduction in the proportion of bradytelic taxa that are identified, rather than a qualitatively false conclusion of linearity and homogeneity. Sepkoski's analyses were mostly of situations more extreme than the ones I used and most of his results have little bearing on mine. I originally excluded some data from any analysis because of related inadequacies. There are many kinds of inaccuracies involved in my analysis, some as yet unpublished, but none known to me seriously affect the predominant linearity of the curves.

The Red Queen's Hypothesis is ecological and does not depend on the linearity of taxonomic survivorship curves which served as midwife. Her domain is less than universal and may be empty: the hypothetico-deductive method is weak, and the sort of facts from which the hypothesis may be deduced are as yet only suggestively available. But the Red Queen is rather flexible, as a runner should be, and her domain may yet include much of evolution.

except for 310 base pairs between the $16 \mathrm{~S}$ and $23 \mathrm{~S}$ rRNA sequences. This means that the internal spacers are different in the two transcription units. When cells are infected by $\phi 80$ rif $^{r}$ $\mathrm{tRNA}_{1}{ }^{\text {Ile }}$ is synthesised, and Lund et al. have confirmed that its gene seems to lie within the spacer region. At least two of the rRNA transcription units of E.coli therefore possess a (different) tRNA sequence; whether the other (approximately 5) units also carry tRNA sequences remains to be established. What function in bacterial protein synthesis might be served by this coupling is not yet clear, although it seems to have the consequence that the tRNA coded with the rRNAs is synthesised in large amounts (both tRNA $_{2}^{\text {Glu }}$ and tRNA ${ }_{1}^{\text {Ile }}$ are among the 